\title{
INEQUALITIES FOR SYMMETRIC MEANS, SYMMETRIC HARMONIC MEANS, AND THEIR APPLICATIONS
}

\author{
Hsu-Tung Ku, Mei-Chin Ku and Xin-Min Zhang
}

In this paper, we establish a number of inequalities involving symmetric means and symmetric harmonic means. We then apply these new inequalities to obtain many geometric inequalities of isoperimetric type for plane polygons.

\section{INTRODUCTION}

Inequalities are basic in pure and applied mathematics which brought together three distinguished mathematicians Hardy, Littlewood and Pólya to publish the famous book "Inequalities" [4]. One of the most well-known inequalities is the arithmetic-geometric mean inequality. Among many important applications of inequalities to other fields, inequalities involving various means, such as symmetric means and symmetric harmonic means are of particular interest. They are used extensively in probability and statistics. In geometry and topology, many basic invariants are also defined in terms of the symmetric means, for instance, the $r$ th mean curvature of a Riemannian submanifold et cetera. Let $\mathbf{R}$ denote the field of real numbers, and $\mathbf{R}_{+}=\{x \in \mathbf{R}: x>0\}$. For $1 \leqslant r \leqslant n$, the $r$ th symmetric mean $P_{n}^{[r]}(\mathbf{a})$ of $\mathbf{a}=\left(a_{1}, a_{2}, \ldots, a_{n}\right) \in \mathbf{R}_{+}^{n}$ is defined by

$$
P_{n}^{[r]}(\mathbf{a})=\left\{\left[\begin{array}{l}
n \\
r
\end{array}\right]^{-1} \sigma_{r}\left(a_{1}, \ldots, a_{n}\right)\right\}^{1 / r}
$$

where

$$
\sigma_{r}\left(a_{1}, \ldots, a_{n}\right)=\sum_{1 \leqslant i_{1}<\ldots<i_{r} \leqslant n} a_{i_{1}} a_{i_{2}} \ldots a_{i_{r}}
$$

is the $r$ th elementary symmetric function of $\mathbf{a}$, and the summation is taken over all possible permutations of $\left(i_{1}, \ldots, i_{r}\right), 1 \leqslant r \leqslant n$. The arithmetic mean $A_{n}(\mathbf{a})$ and the geometric mean $G_{n}(\mathbf{a})$ are simply

$$
A_{n}(\mathbf{a})=P_{n}^{[1]}(\mathbf{a}) \quad \text { and } \quad G_{n}(\mathbf{a})=P_{n}^{[n]}(\mathbf{a}) .
$$

Received 18th December, 1996.

Copyright Clearance Centre, Inc. Serial-fee code: 0004-9729/97 \$A2.00+0.00. 
Set $\mathbf{b}=\left(1 / a_{1}, \ldots, 1 / a_{n}\right)$. The harmonic mean $H_{n}(\mathbf{a})$ of $\mathbf{a}$ is defined as $H_{n}(\mathbf{a})=n / \sigma_{1}(\mathbf{b})$. It is well-known that

$$
A_{n}(\mathbf{a}) \geqslant G_{n}(\mathbf{a}) \geqslant H_{n}(\mathbf{a})
$$

with either equality holding if and only if $a_{1}=a_{2}=\ldots=a_{n}$. Furthermore, we have the following symmetric mean inequality.

Lemma 1.1. (See $[3,7, \mathbf{9}, \mathbf{1 0}]$.) For $1 \leqslant t<r \leqslant n$, and $\mathbf{a} \in \mathbf{R}_{+}^{n}$,

$$
P_{n}^{[t]}(\mathbf{a}) \geqslant P_{n}^{[r]}(\mathbf{a})
$$

with equality holding if and only if $a_{1}=\ldots=a_{n}$.

For $\mathbf{a} \in \mathbf{R}_{+}^{n}$, set $m(\mathbf{a})=\min \left\{a_{i}: 1 \leqslant i \leqslant n\right\}, \bar{m}(\mathbf{a})=\max \left\{a_{i}: 1 \leqslant i \leqslant n\right\}$ and define

$$
e_{n}(\mathbf{a})=4 m(\mathbf{a}) \bar{m}(\mathbf{a}) /\{m(\mathbf{a})+\bar{m}(\mathbf{a})\}^{2} .
$$

By the arithmetic-geometric mean inequality (or Lemma 1.1), we have

$$
e_{n}(\mathbf{a}) \leqslant 1 \text {, with equality if and only if } a_{1}=a_{2}=\ldots=a_{n} .
$$

In terms of $e_{n}(\mathbf{a})$, we can restate a result in $[3$, p.201] as follows in contrast with the inequality $A_{n}(\mathbf{a}) \geqslant H_{n}(\mathbf{a})$.

LEMMA 1.2. (Kantorovich.) For $\mathbf{a} \in \mathbf{R}_{+}^{n}$,

$$
H_{n}(\mathbf{a}) \geqslant e_{n}(\mathbf{a}) A_{n}(\mathbf{a}) \text {. }
$$

Equality holds if and only if $n=2 m$ is even, $a_{1}=\ldots=a_{m}$ and $a_{m+1}=\ldots=a_{2 m}$.

In this paper, we shall generalise the inequality (1) and establish inequalities that reverse the direction of (1) in the sense of (4). In fact, we shall prove the following result.

TheOREM 1.3 Let $1 \leqslant t<r \leqslant n$ and $\mathbf{a} \in \mathbf{R}_{+}^{n}$. Then

$$
P_{n}^{[r]}(\mathbf{a}) \geqslant\left\{e_{n}(\mathbf{a})\right\}^{(r-t) / r} P_{n}^{[t]}(\mathbf{a})
$$

Equality holds if and only if $a_{1}=a_{2}=\ldots=a_{n}$.

We apply these inequalities along with some other inequalities for various means that we have proved earlier in [6] to establish many new interesting geometric isoperimetric inequalities for plane polygons in section 3 .

\section{InEqUALITIES FOR SYMmETRIC AND HARMONIC MEANS}

We shall begin by proving a new inequality which may be viewed as a counterpart of Lemma 1.1.

Theorem 2.1. Let $\mathbf{a} \in \mathbf{R}_{+}^{n}$ and $1 \leqslant t<r<s \leqslant n$.

$$
P_{n}^{[r]}(\mathbf{a}) \geqslant\left\{P_{n}^{[t]}(\mathbf{a})\right\}^{(t(s-r)) /(r(s-t))}\left\{P_{n}^{[s]}(\mathbf{a})\right\}^{(s(r-t)) /(r(s-t))}
$$


Equality holds if and only if $a_{1}=a_{2}=\ldots=a_{n}$.

Proof: First we set

$$
p_{n}^{[r]}(\mathbf{a})=\left\{P_{n}^{[r]}(\mathbf{a})\right\}^{r}=\left[\begin{array}{c}
n \\
r
\end{array}\right]^{-1} \sigma_{r}\left(a_{1}, \ldots, a_{n}\right), \quad 1 \leqslant r \leqslant n .
$$

It is well-known that if $1<r<n$, we have (see $[3,9]$ )

$$
\left\{p_{n}^{[r]}(\mathbf{a})\right\}^{2} \geqslant p_{n}^{[r-1]}(\mathbf{a}) p_{n}^{[r+1]}(\mathbf{a})
$$

with equality if and only if $a_{1}=a_{2}=\ldots=a_{n}$. We claim that

$$
p_{n}^{[s-k]}(\mathbf{a}) \geqslant\left\{p_{n}^{[s-k-1]}(\mathbf{a})\right\}^{k /(k+1)}\left\{p_{n}^{[s]}(\mathbf{a})\right\}^{1 /(k+1)}, \quad k+1<s \leqslant n .
$$

According to (6), (7) is true for $k=1$. Suppose $2 \leqslant r+1 \leqslant n-2$, and (7) is valid for $k=r$. By induction and (6),

$$
\left\{p_{n}^{[s-r-1]}(\mathbf{a})\right\}^{2} \geqslant p_{n}^{[s-r]}(\mathbf{a}) p_{n}^{[s-r-2]}(\mathbf{a}) \geqslant\left\{p_{n}^{[s-r-1]}(\mathbf{a})\right\}^{r /(r+1)}\left\{p_{n}^{[s]}(\mathbf{a})\right\}^{1 /(r+1)} p_{n}^{[s-r-2]}(\mathbf{a})
$$

and so,

$$
\left\{p_{n}^{[s-r-1]}(\mathbf{a})\right\}^{(r+2) /(r+1)} \geqslant p_{n}^{[s-r-2]}(\mathbf{a})\left\{p_{n}^{[s]}(\mathbf{a})\right\}^{1 /(\mathbf{r}+1)} .
$$

Hence (7) holds for $k=r+1$. To prove the theorem, it suffices to prove the following:

$$
p_{n}^{[r]}(\mathbf{a}) \geqslant\left\{p_{n}^{[t]}(\mathbf{a})\right\}^{(s-r) /(s-t)}\left\{p_{n}^{[s]}(\mathbf{a})\right\}^{(r-t) /(s-t)} .
$$

Now, by (7) we obtain

$$
p_{n}^{[2]}(\mathbf{a}) \geqslant\left\{p_{n}^{[1]}(\mathbf{a})\right\}^{(s-2) /(s-1)}\left\{p_{n}^{[s]}(\mathbf{a})\right\}^{1 /(s-1)},
$$

that is, (8) holds for $r=2$. Suppose $3 \leqslant r+1 \leqslant n-1$, and (8) is valid for $k=r$. Then by (7) we get,

$$
\begin{aligned}
p_{n}^{[r+1]}(\mathbf{a}) & \geqslant\left\{p_{n}^{[r]}(\mathbf{a})\right\}^{(s-r-1) /(s-r)}\left\{p_{n}^{[s]}(\mathbf{a})\right\}^{1 /(s-r)} \\
& \geqslant\left[\left\{p_{n}^{[t]}(\mathbf{a})\right\}^{(s-r) /(s-t)}\left\{p_{n}^{[s]}(\mathbf{a})\right\}^{(r-t) /(s-t)}\right]^{(s-r-1) /(s-r)}\left\{p_{n}^{[s]}(\mathbf{a})\right\}^{1 /(s-r)} \\
& =\left\{p_{n}^{[t]}(\mathbf{a})\right\}^{(s-r-1) /(s-t)}\left\{p_{n}^{[s]}(\mathbf{a})\right\}^{(r+1-t) /(s-t)}
\end{aligned}
$$

That is, (8) holds for $k=r+1$. Hence the result follows by induction.

Now, Theorem 1.3 is simply a corollary of the following theorem.

THEOREM 2.2. Let $1 \leqslant t<r \leqslant n$, and $\mathbf{a} \in \mathbf{R}_{+}^{n}$.

$$
P_{n}^{[r]}(\mathbf{a}) \geqslant\left\{e_{n}(\mathbf{a}) A_{n}(\mathbf{a})\right\}^{(r-t) / r}\left\{P_{n}^{[t]}(\mathbf{a})\right\}^{t / r}
$$


with equality if and only if $a_{1}=a_{2}=\ldots=a_{n}$.

Proof: From the definitions,

$$
\left\{G_{n}(\mathbf{a})\right\}^{n}=H_{n}(\mathbf{a})\left\{P_{n}^{[n-1]}(\mathbf{a})\right\}^{n-1} .
$$

Hence, it follows from Lemma 1.2 and Theorem 2.1 that

$$
\begin{aligned}
G_{n}(\mathbf{a}) & \geqslant\left\{e_{n}(\mathbf{a})\right\}^{1 / n}\left\{P_{n}^{[n-1]}(\mathbf{a})\right\}^{(n-1) / n}\left\{A_{n}(\mathbf{a})\right\}^{1 / n} \\
& \geqslant\left\{e_{n}(\mathbf{a})\right\}^{1 / n}\left\{\left[P_{n}^{[t]}(\mathbf{a})\right]^{t /((n-1)(n-t))}\left[G_{n}(\mathbf{a})\right]^{(n(n-1-t)) /((n-1)(n-t))}\right\}^{(n-1) / n}\left\{A_{n}(\mathbf{a})\right\}^{1 / n}
\end{aligned}
$$

Thus,

$$
\left\{G_{n}(\mathbf{a})\right\}^{1 /(n-t)} \geqslant\left\{e_{n}(\mathbf{a})\right\}^{1 / n}\left\{P_{n}^{(t)}(\mathbf{a})\right\}^{t /(n(n-t))}\left\{A_{n}(\mathbf{a})\right\}^{1 / n}
$$

That is,

$$
G_{n}(\mathbf{a}) \geqslant\left\{e_{n}(\mathbf{a}) A_{n}(\mathbf{a})\right\}^{(n-t) / n}\left\{P_{n}^{[t]}(\mathbf{a})\right\}^{t / n} .
$$

Hence (9) holds for $r=n$. If $r<n$, by (11) and Theorem 2.1 we have

$$
\begin{aligned}
P_{n}^{[r]}(\mathbf{a}) & \geqslant\left\{P_{n}^{[t]}(\mathbf{a})\right\}^{(t(n-r)) /(r(n-t))}\left\{G_{n}(\mathbf{a})\right\}^{(n(r-t)) /(r(n-t))} \\
& \geqslant\left\{P_{n}^{[t]}(\mathbf{a})\right\}^{(t(n-r)) /(r(n-t))}\left\{\left[e_{n}(\mathbf{a})\right]^{(n-t) / n}\left[P_{n}^{[t]}(\mathbf{a})\right]^{t / n}\left[A_{n}(\mathbf{a})\right]^{(n-t) / n}\right\}^{(n(r-t)) /(r(n-t))} \\
& =\left\{e_{n}(\mathbf{a}) A_{n}(\mathbf{a})\right\}^{(r-t) / r}\left\{P_{n}^{[t]}(\mathbf{a})\right\}^{t / r}
\end{aligned}
$$

Recall that if we set $\mathbf{b}=\left(1 / a_{1}, \ldots, 1 / a_{n}\right)$, then $H_{n}(\mathbf{a})=1 / P_{n}^{[1]}(\mathbf{b})$. Hence we can define the $r$ th symmetric harmonic mean $H_{n, r}(\mathbf{a})$ of $\mathbf{a} \in \mathbf{R}_{+}^{n}$ by

$$
H_{n, r}(\mathbf{a})=1 /\left(P_{n}^{[r]}(\mathbf{b})\right), \quad 1 \leqslant r \leqslant n .
$$

Observe that $H_{n}(\mathbf{a})=H_{n, 1}(\mathbf{a})$ and $H_{n, n}(\mathbf{a})=G_{n}(\mathbf{a})$. As an immediate corollary to Lemma 1.1 and Theorem 1.3 we have the following inequalities.

THEOREM 2.3. Let $\mathbf{a} \in \mathbf{R}_{+}^{n}$ and $1 \leqslant t<r \leqslant n$. Then

(a) $H_{n, t}(\mathbf{a}) \leqslant H_{n, r}(\mathbf{a})$.

(b) $H_{n, t}(\mathbf{a}) \geqslant\left\{e_{n}(\mathbf{a})\right\}^{(r-t) / r} H_{n, r}(\mathbf{a})$.

Equality holds in either (a) or (b) if and only if $a_{1}=a_{2}=\ldots=a_{n}$.

\section{Geometric Isoperimetric Inequalities}

As pointed out by Pólya in his excellent book Induction and Analogy in Mathematics [13], isoperimetric inequalities and inequalities for means share a lot of common properties. In this section, we shall apply the results about various means in previous sections and some other inequalities that we have obtained earlier in [6] to establish geometric 
inequalities for plane polygons. We are able to obtain uncountably many new isoperimetric inequalities which are not only interesting in geometry, but also very important in analysis, as well as in mathematical physics $[2,11,12,14,15]$. Let $\mathcal{P}_{n}$ be an $n$ sided polygon in the plane and $a_{1}, a_{2}, \ldots, a_{n}$ denote the lengths of its sides. Denote by $A\left(\mathcal{P}_{n}\right)$ and $L\left(\mathcal{P}_{n}\right)=\sum_{i=1}^{n} a_{i}$, the area and the perimeter of $\mathcal{P}_{n}$ respectively. The well-known classical isoperimetric inequality asserts that $[12$, p.1209]:

$$
L^{2}\left(\mathcal{P}_{n}\right) / A\left(\mathcal{P}_{n}\right) \geqslant 4 d_{n}, \quad \text { where } \quad d_{n}=n \tan (\pi / n) .
$$

Equality holds if and only if $\mathcal{P}_{n}$ is regular.

Set $\alpha=\left(\alpha_{1}, \alpha_{2}, \ldots, \alpha_{n}\right) \in \mathbf{R}_{+}^{n}, \alpha_{i}=L\left(\mathcal{P}_{n}\right)-2 a_{i}, 1 \leqslant i \leqslant n$. Then we have

$$
L\left(P_{n}\right)=\frac{n}{n-2} P_{n}^{[1]}(\alpha) \text {. }
$$

For $n=3$ and $n=4$, the famous Heron formula for a triangle and Brahmagupta formula for a quadrilateral can be expressed as follows $[5,7,8]$ :

$$
\begin{gathered}
\left\{\frac{n}{n-2}\left[A_{n}(\alpha)\right]^{(n-2) /(2(n-1))}\left[G_{n}(\alpha)\right]^{n /(2(n-1))}\right\}^{2}=4 d_{n} A\left(\mathcal{P}_{n}\right), \quad(n=3) . \\
\left\{\frac{n}{n-2} P_{n}^{[n]}(\alpha)\right\}^{2} \geqslant 4 d_{n} A\left(\mathcal{P}_{n}\right), \quad(n=4) .
\end{gathered}
$$

The equality in (15) holds if and only if $\mathcal{P}_{4}$ is a cyclic quadrilateral, that is, it can be inscribed in a circle. It is a well-known fact that "Of all $n$-sided plane polygons with given $n$ sides, the cyclic polygon encloses the largest area" $[\mathbf{5}, \mathbf{8}]$. Since we are mainly concerned with isoperimetric problem for polygons, from now on, all polygons will be assumed to be cyclic unless otherwise specified. For the simplicity of statement, it is convenient to use $\alpha$ instead of $\mathbf{a} \in \mathbf{R}_{+}^{n}$ when we discuss isoperimetric inequalities for polygons by virtue of inequalities for means. Formulas (13), (14), and (15) also motivate us to introduce the following new geometric invariants.

DEFINITION 3.1: The $r$ th symmetric perimeter $\mathcal{L}_{n}^{[r]}\left(\mathcal{P}_{n}\right)$ and the $r$ th symmetric harmonic perimeter $\mathcal{L}_{n, r}\left(\mathcal{P}_{n}\right)$ of $\mathcal{P}_{n}, 1 \leqslant r \leqslant n$, are defined respectively by

$$
\mathcal{L}_{n}^{[r]}\left(\mathcal{P}_{n}\right)=\frac{n}{n-2} P_{n}^{[r]}(\alpha), \quad \text { and } \quad \mathcal{L}_{n, r}\left(\mathcal{P}_{n}\right)=\frac{n}{n-2} H_{n, r}(\alpha) .
$$

Set $\mathcal{H}_{n}\left(\mathcal{P}_{n}\right)=\mathcal{L}_{n, 1}\left(\mathcal{P}_{n}\right)$ and call it the harmonic perimeter of $\mathcal{P}_{n}$. By Lemma 1.1 and Theorem 2.3 (a) we have

$$
L\left(\mathcal{P}_{n}\right)=\mathcal{L}_{n}^{[1]}\left(\mathcal{P}_{n}\right) \geqslant \mathcal{L}_{n}^{[2]}\left(\mathcal{P}_{n}\right) \geqslant \ldots \geqslant \mathcal{L}_{n}^{[n]}\left(\mathcal{P}_{n}\right)=\mathcal{L}_{n, n}\left(\mathcal{P}_{n}\right)
$$

and

$$
\mathcal{L}_{n, n}\left(\mathcal{P}_{n}\right) \geqslant \mathcal{L}_{n, n-1}\left(\mathcal{P}_{n}\right) \geqslant \ldots \geqslant \mathcal{L}_{n, 1}\left(\mathcal{P}_{n}\right)=\mathcal{H}_{n}\left(\mathcal{P}_{n}\right)
$$

Now, let us set $e_{n}\left(\mathcal{P}_{n}\right)=e_{n}(\alpha)$. By $(3), e_{n}\left(\mathcal{P}_{n}\right) \leqslant 1$ with equality if and only if $\mathcal{P}_{n}$ is regular. We present the following generalisation and variations of (12).

THEOREM 3.2. For $r=1,2, \ldots, n$, we have 
(a) $\left\{\mathcal{L}_{n}^{[r]}\left(\mathcal{P}_{n}\right)\right\}^{2} / A\left(\mathcal{P}_{n}\right) \geqslant 4 d_{n}\left\{e_{n}\left(\mathcal{P}_{n}\right)\right\}^{(2(r-1)) / r}$
(b) $\left\{\mathcal{L}_{n, r}\left(\mathcal{P}_{n}\right)\right\}^{2} / A\left(\mathcal{P}_{n}\right) \geqslant 4 d_{n}\left\{e_{n}\left(\mathcal{P}_{n}\right)\right\}^{(2(2 n-r-1)) / n}$

In particular,

$$
\left\{\mathcal{H}_{n}\left(\mathcal{P}_{n}\right)\right\}^{2} / A\left(\mathcal{P}_{n}\right) \geqslant 4 d_{n}\left\{e_{n}\left(\mathcal{P}_{n}\right)\right\}^{(4(n-1)) / n} .
$$

Equality holds in (a) (respectively (b)) if and only if $\mathcal{P}_{n}$ is regular.

Proof: Apply Theorem 1.3 to $\alpha \in \mathbf{R}_{+}^{n}$.

Notice that if $r=1$, (a) is simply the inequality (12).

Next, we shall briefly review the so-called generalised power means and their inequalities that we have obtained in [6]. Then we shall be able to establish a family of isoperimetric inequalities that are much more general than Theorem 3.2. Let $f_{i}: \mathbf{R}_{+}^{n} \longrightarrow \mathbf{R}_{+}$, $1 \leqslant i \leqslant m$, be distinct functions, and $\mathbf{f}=\left(f_{1}, f_{2}, \ldots f_{m}\right)$. Let $w_{i}>0,1 \leqslant i \leqslant m$, and $\Delta(\mathbf{w})=\Delta\left(w_{1}, \ldots, w_{m}\right)$ be the $(m-1)$-simplex in $\mathbf{R}^{m}$ with vertices

$$
\mathbf{W}_{i}=\left(0, \ldots, 0,1 / w_{i}, 0, \ldots, 0\right) \quad \text { where } 1 / w_{i} \text { is the } i \text { th coordinate, } i=1, \ldots, m \text {. }
$$

Thus, if $\mathbf{x}=\left(x_{1}, \ldots, x_{m}\right) \in \Delta(\mathbf{w})$, then $\sum_{i=1}^{m} w_{i} x_{i}=1$. For $\mathbf{x} \in \Delta(\mathbf{w}), \mathbf{a} \in \mathbf{R}_{+}^{n}$, and $r \geqslant 0$, we have defined the generalised power mean $L_{n, m}^{[r]}[\mathbf{f} ; \mathbf{x} ; \mathbf{w}](\mathbf{a})$ in $[\mathbf{6}]$ by

$$
L_{n, m}^{[r]}[\mathbf{f} ; \mathbf{x} ; \mathbf{w}](\mathbf{a})= \begin{cases}\prod_{i=1}^{m}\left\{f_{i}(\mathbf{a})\right\}^{w_{i} x_{i}} & \text { if } r=0 \\ \left\{\sum_{i=1}^{m} w_{i} x_{i}\left[f_{i}(\mathbf{a})\right]^{r}\right\}^{1 / r} & \text { if } r>0\end{cases}
$$

Moreover, if $0 \leqslant t<r, \mathbf{x} \in \Delta(\mathbf{w})$, the following inequality generalises the ordinary power mean inequality, and was proved in [6, Theorem 2.5(a)]:

$$
L_{n, m}^{[t]}[\mathbf{f} ; \mathbf{x} ; \mathbf{w}](\mathbf{a}) \leqslant L_{n, m}^{[r]}[\mathbf{f} ; \mathbf{x} ; \mathbf{w}](\mathbf{a}), \quad \mathbf{a} \in \mathbf{R}_{+}^{n} \text {. }
$$

Equality holds if and only if $a_{1}=a_{2}=\ldots=a_{n}$.

We now can define the mixed symmetric perimeter $\mathcal{L}_{n}^{[r]}[\mathbf{x} ; \mathbf{w}]\left(\mathcal{P}_{n}\right)$ and the mixed symmetric harmonic perimeter $\mathcal{L}_{n, r}[\mathbf{x} ; \mathbf{w}]\left(\mathcal{P}_{n}\right)$ for $\mathcal{P}_{n}$ with $\mathbf{w} \in \mathbf{R}_{+}^{n}$ and $\mathbf{x} \in \Delta(\mathbf{w})$, $0 \leqslant r \leqslant n$, as following:

(19) $\mathcal{L}_{n}^{[r]}[\mathbf{x} ; \mathbf{w}]\left(\mathcal{P}_{n}\right)=\frac{n}{n-2} L_{n, n}^{[r]}[\mathbf{f} ; \mathbf{x} ; \mathbf{w}](\alpha), \quad$ where $\quad f_{i}(\alpha)=P_{n}^{[i]}(\alpha), \quad 1 \leqslant i \leqslant n$.

$(20) \mathcal{L}_{n, r}[\mathbf{x} ; \mathbf{w}]\left(\mathcal{P}_{n}\right)=\frac{n}{n-2} L_{n, n}^{[r]}[\mathbf{f} ; \mathbf{x} ; \mathbf{w}](\alpha), \quad$ where $\quad f_{i}(\alpha)=H_{n, i}(\alpha), \quad 1 \leqslant i \leqslant n$.

Clearly, if $\mathbf{x}=\mathbf{W}_{i}$,

$$
\mathcal{L}_{n}^{[r]}[\mathbf{x} ; \mathbf{w}]\left(\mathcal{P}_{n}\right)=\mathcal{L}_{n}^{[\mathbf{r}]}\left(\mathcal{P}_{n}\right), \quad \text { and } \quad \mathcal{L}_{n, r}[\mathbf{x} ; \mathbf{w}]\left(\mathcal{P}_{n}\right)=\mathcal{L}_{n, r}\left(\mathcal{P}_{n}\right)
$$


In [6], we introduced a dominance relation $\succ$ on $\Delta(\mathbf{w})$ as follows: for $\mathbf{x}, \mathbf{x}^{\prime} \in \Delta(\mathbf{w})$, $\mathbf{x} \succ \mathbf{x}^{\prime}$ if $\mathbf{x}=\mathbf{x}^{\prime}$, or there exists an integer $k, 1 \leqslant k<m$ such that $x_{i} \geqslant x_{i}^{\prime}$ for $1 \leqslant i \leqslant k$, $x_{k+1}<x_{k+1}^{\prime}$; and $x_{i} \leqslant x_{i}^{\prime}$ for $k+2 \leqslant i \leqslant m$, if $k+2 \leqslant m$. We proved a fundamental inequality for generalised power means [6, Theorem 2.5(b)]. That is, if $\mathbf{x}, \mathbf{x}^{\prime} \in \Delta(\mathbf{w})$ and $\mathbf{x} \succ \mathbf{x}^{\prime}$, then

$$
L_{n, m}^{[r]}[\mathbf{f} ; \mathbf{x} ; \mathbf{w}](\mathbf{a}) \geqslant L_{n, m}^{[r]}\left[\mathbf{f} ; \mathbf{x}^{\prime} ; \mathbf{w}\right](\mathbf{a}) .
$$

As direct consequences of this result, we have the following inequalities for the mixed symmetric perimeters.

Theroem 3.3. Suppose $\mathbf{x} \succ \mathbf{x}^{\prime} ; \mathbf{x}, \mathbf{x}^{\prime} \in \triangle(\mathbf{w}), \mathbf{w} \in \mathbf{R}_{+}^{n}$, and $0 \leqslant r \leqslant n$. Then

(a) $\mathcal{L}_{n}^{[r]}[\mathbf{x} ; \mathbf{w}]\left(\mathcal{P}_{n}\right) \geqslant \mathcal{L}_{n}^{[r]}\left[\mathbf{x}^{\prime} ; \mathbf{w}\right]\left(\mathcal{P}_{n}\right)$

(b) $\mathcal{L}_{n, r}[\mathbf{x} ; \mathbf{w}]\left(\mathcal{P}_{n}\right) \geqslant \mathcal{L}_{n, r}\left[\mathbf{x}^{\prime} ; \mathbf{w}\right]\left(\mathcal{P}_{n}\right)$.

If $\mathbf{x} \neq \mathbf{x}^{\prime}$, then equality holds in (a) (respectively (b)) if and only if $\mathcal{P}_{n}$ is regular.

By using these new geometric quantities of $\mathcal{P}_{n}$, Theorem 3.2 can be generalised even further.

THEOREM 3.4. For $\mathbf{w} \in \mathbf{R}_{+}^{n}$, and $\mathbf{x} \in \triangle(\mathbf{w})$, we have

$$
\begin{aligned}
& \text { (a) }\left\{\mathcal{L}_{n}^{[r]}[\mathbf{x} ; \mathbf{w}]\left(\mathcal{P}_{n}\right)\right\}^{2} / A\left(\mathcal{P}_{n}\right) \geqslant 4 d_{n}\left\{e_{n}\left(\mathcal{P}_{n}\right)\right\}^{2-2 \sum_{i=1}^{m} w_{i} x_{i} / i} . \\
& \text { (b) }\left\{\mathcal{L}_{n, r}[\mathbf{x} ; \mathbf{w}]\left(\mathcal{P}_{n}\right)\right\}^{2} / A\left(\mathcal{P}_{n}\right) \geqslant 4 d_{n}\left\{e_{n}\left(\mathcal{P}_{n}\right)\right\}^{4-(2 / n)-(2 / n) \sum_{i=1}^{m} i w_{i} x_{i}} .
\end{aligned}
$$

Equality holds in (a) or (b) if and only if $\mathcal{P}_{n}$ is regular.

Proof: (a) By (18) and Theorem 3.2 (a),

$$
\begin{aligned}
\left\{\mathcal{L}_{n}^{[r]}[\mathbf{x} ; \mathbf{w}]\left(\mathcal{P}_{n}\right)\right\}^{2} / A\left(\mathcal{P}_{n}\right) & \geqslant\left\{\mathcal{L}_{n}^{[0]}[\mathbf{x} ; \mathbf{w}]\left(\mathcal{P}_{n}\right)\right\}^{2} / A\left(\mathcal{P}_{n}\right) \\
& =\prod_{i=1}^{n}\left\{\left[\mathcal{L}_{n}^{[i]}\left(\mathcal{P}_{n}\right)\right]^{2} / A\left(\mathcal{P}_{n}\right)\right\}^{w_{i} x_{i}} \\
& \geqslant 4 d_{n}\left\{e_{n}\left(\mathcal{P}_{n}\right)\right\}^{2-2 \sum_{i=1}^{m} w_{i} x_{i} / i}
\end{aligned}
$$

(b) Similar.

We can restate (14) and (15) respectively as

$$
\begin{aligned}
& \left\{\mathcal{L}_{3}^{[2]}\left(\mathcal{P}_{3}\right)\right\}^{2} / A\left(\mathcal{P}_{3}\right)=4 d_{3}, \\
& \left\{\mathcal{L}_{4}^{[4]}\left(\mathcal{P}_{4}\right)\right\}^{2} / A\left(\mathcal{P}_{4}\right) \geqslant 4 d_{4} .
\end{aligned}
$$

Thus, we may ask the following questions:

QUESTION 3.5. Can one improve Theorem 3.4 to obtain the following stronger inequalities:

$$
\left\{\mathcal{L}_{n}^{[r]}[\mathbf{x} ; \mathbf{w}]\left(\mathcal{P}_{n}\right)\right\}^{2} / A\left(\mathcal{P}_{n}\right) \geqslant 4 d_{n}
$$




$$
\left\{\mathcal{L}_{n, r}[\mathbf{x} ; \mathbf{w}]\left(\mathcal{P}_{n}\right)\right\}^{2} / A\left(\mathcal{P}_{n}\right) \geqslant 4 d_{n}
$$

with equality in either (21) or (22) if and only if $\mathcal{P}_{n}$ is regular?

Observe that from $(16),(17),(19),(20)$, together with Theorem 3.3 , the mixed symmetric perimeters in (21) and (22) are all smaller than or equal to the ordinary perimeter $L\left(\mathcal{P}_{n}\right)$ of $\mathcal{P}_{n}$. For example,

$$
L^{2}\left(\mathcal{P}_{n}\right)-\left\{\mathcal{L}_{n}^{[r]}[\mathbf{x} ; \mathbf{w}]\left(\mathcal{P}_{n}\right)\right\}^{2}=B\left(\mathcal{P}_{n}\right) \geqslant 0, \quad \mathbf{w} \in \mathbf{R}_{+}^{n}, \mathbf{x} \in \Delta(\mathbf{w})
$$

with equality holding if and only if $\mathcal{P}_{n}$ is regular. If the answers to the questions were to be affirmative, one of its significances is that we would have a large family of the following Bonnesen-style inequalities for plane polygons [16]

$$
L^{2}\left(\mathcal{P}_{n}\right)-4 d_{n} A\left(\mathcal{P}_{n}\right) \geqslant B\left(\mathcal{P}_{n}\right)
$$

As a matter of fact, a class of cyclic polygons satisfying (21) and (22) does exist. Let $\mathcal{P}_{n}=\mathcal{P}_{m, m}(n=2 m)$ denote cyclic $2 m$-gons with $m$ sides of length $b$ and remaining $m$ sides of length $c$. Set

$$
\begin{gathered}
q=\frac{(b-c)^{2}}{2\left[\left(b^{2}+c^{2}\right) \cos (\pi / m)+2 b c\right]}, \quad \alpha(m)=1+q\left(1-\cos \frac{\pi}{m}\right), \quad \text { and } \\
\beta(m)=\alpha(m)-\frac{q(1+\cos (\pi / m))}{(m-1)^{2}} .
\end{gathered}
$$

If $m \geqslant 3$, a simple calculation using $\cos \theta=\sum_{i=0}^{\infty}(-1)^{i}\left(\theta^{2 i} /(2 i) !\right)$ yields

$$
1-\cos \frac{\pi}{m}>\frac{1+\cos (\pi / m)}{(m-1)^{2}}, \quad m \geqslant 3
$$

Hence, we have

$$
\alpha(m)>\beta(m)>1 \quad \text { if } \quad m \geqslant 3 .
$$

LEMma 3.6. Let $\mathcal{P}_{n}=\mathcal{P}_{m, m}$. Then

(a) $\left\{\mathcal{L}_{n}^{[1]}\left(\mathcal{P}_{n}\right)\right\}^{2} / A\left(\mathcal{P}_{n}\right)=4 d_{n} \alpha(m)$.

(b) $\left\{\mathcal{L}_{n}^{[n]}\left(\mathcal{P}_{n}\right)\right\}^{2} / A\left(\mathcal{P}_{n}\right)=4 d_{n} \beta(m)$.

(c) $\left\{\mathcal{H}_{n}\left(\mathcal{P}_{n}\right)\right\}^{2} / A\left(\mathcal{P}_{n}\right)=4 d_{n}\{\beta(m)\}^{2} / \alpha(m)$.

ProOF: In [8], Macnab has proved that

$$
A\left(\mathcal{P}_{n}\right)=\frac{m}{4 \sin (\pi / m)}\left[\left(b^{2}+c^{2}\right) \cos \frac{\pi}{m}+2 b c\right] .
$$

Hence the results follow directly from the definitions. 
LEммA 3.7. If $m \geqslant 10$, then $\{\beta(m)\}^{2} \geqslant \alpha(m)$.

PROOF: Set

$$
\phi(m)=\frac{-1+\cos \frac{\pi}{m}+\frac{2(1+\cos (\pi / m))}{(m-1)^{2}}}{\left[1-\cos \frac{\pi}{m}-\frac{(1+\cos (\pi / m))}{(m-1)^{2}}\right]^{2}} .
$$

Obviously, $\{\beta(m)\}^{2} / \alpha(m) \geqslant 1$ if and only if $q \geqslant \phi(m)$. Since $q>0$, it suffices to verify that $\phi(m)<0$ if $m \geqslant 10$. But

$$
\phi(m)<0 \Longleftrightarrow \cos \frac{\pi}{m}<\frac{m^{2}-2 m-1}{m^{2}-2 m+3}
$$

If $m \geqslant 10$

$$
\frac{4}{m^{2}-2 m+3}+\frac{1}{4 !}\left(\frac{\pi}{m}\right)^{4}<\frac{\pi^{2}}{2 m^{2}}
$$

and so,

$$
\cos \frac{\pi}{m}<1-\frac{1}{2 !}\left(\frac{\pi}{m}\right)^{2}+\frac{1}{4 !}\left(\frac{\pi}{m}\right)^{4} \leqslant 1-\frac{4}{m^{2}-2 m+3}=\frac{m^{2}-2 m-1}{m^{2}-2 m+3} .
$$

This proves the Lemma.

Now, the answer to Question 3.5 is affirmative for $\mathcal{P}_{m, m}$ if $m \geqslant 10$. In fact, we have the following result.

Theorem 3.8. Let $m \geqslant 10,0 \leqslant r \leqslant n$, w $\in \mathbf{R}_{+}^{n}$, and $\mathbf{x} \in \triangle(\mathbf{w})$. Then the isoperimetric inequalities (21) and (22) hold for polygon $\mathcal{P}_{n}=\mathcal{P}_{m, m}$.

Proof: By (16), (17) and Theorem 3.3, it suffices to show that

$$
\left\{\mathcal{H}_{n}\left(\mathcal{P}_{n}\right)\right\}^{2} / A\left(\mathcal{P}_{n}\right) \geqslant 4 d_{n}
$$

with equality if and only if $\mathcal{P}_{n}$ is regular. As a matter of fact, from Lemmas 3.6, 3.7 we see that it is certainly true.

REMARK. A special case of inequality (21) in Theorem 3.8 for $r=0$ was proved in [7]. For $\mathcal{P}_{n}=\mathcal{P}_{m, m}, m \geqslant 10$, we can improve inequalities (21) and (22).

TheOREM 3.9. Let $m \geqslant 10,0 \leqslant r \leqslant n$, w $\in \mathbf{R}_{+}^{n}, \mathbf{x} \in \triangle(\mathbf{w})$, and $\mathcal{P}_{n}=\mathcal{P}_{m, m}$.

$$
\begin{aligned}
& \text { (a) }\left\{\mathcal{L}_{n}^{[r]}[\mathbf{x} ; \mathbf{w}]\left(\mathcal{P}_{n}\right)\right\}^{2} / A\left(\mathcal{P}_{n}\right) \geqslant 4 d_{n}[\alpha(m)]^{\sum_{i=1}^{n} w_{i} x_{i}((n-2) i+n) /(i(n-1))} . \\
& \text { (b) }\left\{\mathcal{L}_{n, r}[\mathbf{x} ; \mathbf{w}]\left(\mathcal{P}_{n}\right)\right\}^{2} / A\left(\mathcal{P}_{n}\right) \geqslant 4 d_{n}[\alpha(m)]^{2-n \sum_{i=1}^{n}\left(w_{i} x_{i}\right) / i} .
\end{aligned}
$$

Equality holds in (a) or (b) if and only if $\mathcal{P}_{n}$ is regular.

Proof: (a) By Lemma 3.6(b) and Lemma 3.7 we have

$$
\left\{\mathcal{L}_{n}^{[n]}\left(\mathcal{P}_{n}\right)\right\}^{2} / A\left(\mathcal{P}_{n}\right) \geqslant 4 d_{n}\{\alpha(m)\}^{1 / 2}
$$


It follows from (4) that

$$
\left\{\mathcal{L}_{n}^{[r]}\left(\mathcal{P}_{n}\right)\right\}^{2} / A\left(\mathcal{P}_{n}\right) \geqslant\left\{\mathcal{L}_{n}^{[1]}\left(\mathcal{P}_{n}\right)\right\}^{2(n-r) / r(n-1)}\left\{\mathcal{L}_{n}^{[n]}\left(\mathcal{P}_{n}\right)\right\}^{2 n(r-1) / r(n-1)} / A\left(\mathcal{P}_{n}\right)
$$

hence by Lemma 3.6 and (27), we have

$$
\left\{\mathcal{L}_{n}^{[r]}\left(\mathcal{P}_{n}\right)\right\}^{2} / A\left(\mathcal{P}_{n}\right) \geqslant 4 d_{n}\{\alpha(m)\}^{(r(n-2)+n) / r(n-1)}
$$

Repeating the proof of Theorem 3.4 using (28) and (18) completes the proof.

(b) From the definition, we can see that

$$
\left\{\mathcal{L}_{n}^{[n]}\left(\mathcal{P}_{n}\right)\right\}^{n}=\left\{\mathcal{L}_{n, r}\left(\mathcal{P}_{n}\right)\right\}^{r}\left\{\mathcal{L}_{n}^{[n-r]}\left(\mathcal{P}_{n}\right)\right\}^{n-r}
$$

By Lemma 3.6, $\left\{\mathcal{L}_{n}^{[n]}\left(\mathcal{P}_{n}\right)\right\}^{2}=\mathcal{L}_{n}^{[1]}\left(\mathcal{P}_{n}\right) \mathcal{H}_{n}\left(\mathcal{P}_{n}\right)$, and $\mathcal{L}_{n}^{[1]}\left(\mathcal{P}_{n}\right) \geqslant \mathcal{L}_{n}^{[n-1]}\left(\mathcal{P}_{n}\right)$ by Lemma 1.1, hence

$$
\begin{aligned}
\left\{\mathcal{L}_{n, r}\left(\mathcal{P}_{n}\right)\right\}^{2} / A\left(\mathcal{P}_{n}\right) & =\left\{\mathcal{L}_{n}^{[n]}\left(\mathcal{P}_{n}\right)\right\}^{2 n / r} /\left\{\mathcal{L}_{n}^{[n-r]}\left(\mathcal{P}_{n}\right)\right\}^{2(n-r) / r} A\left(\mathcal{P}_{n}\right) \\
& \geqslant\left\{\mathcal{L}_{n}^{[1]}\left(\mathcal{P}_{n}\right) \mathcal{H}_{n}\left(\mathcal{P}_{n}\right)\right\}^{n / r} /\left\{\mathcal{L}_{n}^{[1]}\left(\mathcal{P}_{n}\right)\right\}^{2(n-r) / r} A\left(\mathcal{P}_{n}\right) \\
& =\left\{\mathcal{H}_{n}\left(\mathcal{P}_{n}\right)\right\}^{n / r}\left\{\mathcal{L}_{n}^{[1]}\left(\mathcal{P}_{n}\right)\right\}^{2-n / r} / A\left(\mathcal{P}_{n}\right)
\end{aligned}
$$

Thus, by (26), we get

$$
\left\{\mathcal{L}_{n, n}\left(\mathcal{P}_{n}\right)\right\}^{2} / A\left(\mathcal{P}_{n}\right) \geqslant 4 d_{n}\{\alpha(m)\}^{2-n / r}
$$

From (30) and (18),

$$
\left\{\mathcal{L}_{n, r}[\mathbf{x} ; \mathbf{w}]\left(\mathcal{P}_{n}\right)\right\}^{2} / A\left(\mathcal{P}_{n}\right) \geqslant\left\{\frac{n}{n-2} L_{n}^{[0]}[\mathbf{f} ; \mathbf{x} ; \mathbf{w}](\alpha)\right\}^{2} / A\left(\mathcal{P}_{n}\right) \geqslant 4 d_{n}\{\alpha(m)\}^{2-n \sum_{i=1}^{n}\left(w_{i} x_{i}\right) / i}
$$

The isoperimetric inequalities (21) and (22) hold for some other types of polygons. For instance, if $\mathcal{P}_{21}=\mathcal{P}_{10,11}$, that is, $a_{1}=a_{2}=\ldots=a_{10}$ and $a_{11}=a_{12}=\ldots=a_{21}$, then (26) still holds. However, inequality (26) is not always true due to the following results.

Theorem 3.10. Let $\mathcal{P}_{n}=\mathcal{P}_{m, m}$.

(a) If $3 \leqslant m \leqslant 8$, then

$$
\left\{\mathcal{H}_{n}\left(\mathcal{P}_{n}\right)\right\}^{2} / A\left(\mathcal{P}_{n}\right) \leqslant 4 d_{n},
$$

with equality holding if and only if $\mathcal{P}_{n}$ is regular.

(b) For $m=9$, we have
(i) $\left\{\mathcal{H}_{18}\left(\mathcal{P}_{18}\right)\right\}^{2} / A\left(\mathcal{P}_{18}\right) \leqslant 4 d_{18}, \quad$ if $\quad q \leqslant \phi(9)$.
(ii) $\left\{\mathcal{H}_{18}\left(\mathcal{P}_{18}\right)\right\}^{2} / A\left(\mathcal{P}_{18}\right) \geqslant 4 d_{18}, \quad$ if $\quad q \geqslant \phi(9)$. 
Equality holds if and only if $\mathcal{P}_{18}$ is regular and $q=\phi(9)$.

Proof: (a) Since $\{\beta(m)\}^{2} \leqslant \alpha(m)$ for $3 \leqslant m \leqslant 8$ because $\phi(3)=16, \phi(4)=$ $8.116 \ldots, \phi(5)=5.788 \ldots, \phi(6)=4.348 \ldots, \phi(7)=3.078 \ldots$, and $\phi(8)=1.7705 \ldots$

(b) $\phi(9)=0.3422 \ldots$, and $\{\beta(m)\}^{2} \leqslant \alpha(m)$ if and only if $q \leqslant \phi(9)$.

One of the importances of the geometric isoperimetric inequalities is that they are closely related to the eigenvalue problems for the Laplace operator $[12,14,15]$. To conclude this paper, we shall point out that our new geometric findings can be used to reformulate the famous Pólya conjecture for polygons, and prove the conjecture in some special cases.

\section{REFERENCES}

[1] E.F. Beckenbach and R. Bellman, Inequalities (Springer-Verlag, Berlin, Heidelberg, New York, 1965).

[2] R. Brooks and P. Wakesman, 'The first eigenvalue of a scalene triangle', Proc. Amer. Math. Soc. 100 (1987), 175-182..

[3] P.S. Bullen, D.S. Mitrinović and P.M. Vasić, Means and their inequalities (Reidel, Dordrecht, 1988).

[4] G. Hardy, J.E. Littlewood and G. Pólya, Inequalities (Cambridge University Press, Cambridge, New York, 1951).

[5] M.D. Kazarinoff, Geometric inequalities, New Math. Library (Random House, New York, 1961).

[6] H.T. Ku, M.C. Ku and X.M. Zhang, 'Generalized power means and interpolating inequalities', (preprint).

[7] H.T. Ku, M.C. Ku and X.M. Zhang, 'Analytic and geometric isoperimetric inequalities', J. Geom. 53 (1995), 100-121.

[8] D.S. Macnab, 'Cyclic polygons and related questions', Math. Gaz. 65 (1981), 22-28.

[9] D.S. Mitrinović, Analytic inequalities (Springer-Verlag, Berlin, Heidelberg, New York, 1970).

[10] D.S. Mitrinović, J.E. Pečarić and A.M. Fink, Classical and new inequalities in analysis (Kluwer Academic Publishers, Dordrecht, Boston, London, 1993).

[11] D.S. Mitrinović, J.E. Pečarić and V. Volenec, Recent advances in geometric inequalities (Kluwer Academic Publishers, Dordrecht, Boston, London, 1989).

[12] R. Osserman, 'The isoperimetric inequalities', Bull. Amer. Math. Soc. 84 (1978), 1182-1238.

[13] G. Pólya, Mathematics and plausible reasoning I, Induction and Analogy in Mathematics (Princeton University Press, Princeton, NJ, 1954.).

[14] G. Pólya, 'On the eigenvalues of vibrating membrances', London Math. Soc. 11 (1961), $414-433$.

[15] G. Pólya and G. Szegö, Isoperimetric inequalities in mathematical physics, Annals of Mathematics 27 (Princeton, NJ, 1951).

[16] X.M. Zhang, 'Bonnesen-style inequalities and pseudo-perimeters for polygons', J. Geom. (to appear). 
Department of Mathematics and Statistics University of Massachusetts at Amherst Amherst, MA 01003

United States of America

e-mail: hku@math.umass.edu

meiku@math.umass.edu
Department of Mathematics and Statistics University of South Alabama

Mobile, AL 36688

United States of America

e-mail: zhang@mathstat.usouthal.edu 